\title{
Factors influencing access to education, decision making, and receipt of preferred dialysis modality in unplanned dialysis start patients
}

This article was published in the following Dove Press journal:

Patient Preference and Adherence

2 November 2016

Number of times this article has been viewed

\author{
Anna Machowska' \\ Mark Dominik Alscher ${ }^{2}$ \\ Satyanarayana Reddy Vanga ${ }^{3}$ \\ Michael Koch ${ }^{4}$ \\ Michael Aarup 5 \\ Abdul Rashid Qureshi' \\ Bengt Lindholm' \\ Peter A Rutherford ${ }^{6}$ \\ 'Division of Renal Medicine and \\ Baxter Novum, Department of \\ Clinical Science, Intervention, and \\ Technology, Karolinska Institutet, \\ Stockholm, Sweden; ${ }^{2}$ Division of \\ Nephrology, Department of Internal \\ Medicine, Robert-Bosch-Krankenhaus, \\ Stuttgart, Germany; ${ }^{3}$ Department of \\ Renal Medicine, University Hospital \\ of North Staffordshire, Stoke-on- \\ Trent, Staffordshire, UK; ${ }^{4}$ Center \\ of Nephrology, Nephrologisches \\ Zentrum, Mettmann, Germany; \\ ${ }^{5}$ Department of Nephrology, Odense \\ University Hospital, Odense, \\ Denmark; 'Baxter Healthcare SA, \\ Zurich, Switzerland
}

Objectives: Unplanned dialysis start (UPS) leads to worse clinical outcomes than planned start, and only a minority of patients ever receive education on this topic and are able to make a modality choice, particularly for home dialysis. This study aimed to determine the predictive factors for patients receiving education, making a decision, and receiving their preferred modality choice in UPS patients following a UPS educational program (UPS-EP).

Methods: The Offering Patients Therapy Options in Unplanned Start (OPTiONS) study examined the impact of the implementation of a specific UPS-EP, including decision support tools and pathway improvement on dialysis modality choice. Linear regression models were used to examine the factors predicting three key steps: referral and receipt of UPS-EP, modality decision making, and actual delivery of preferred modality choice. A simple economic assessment was performed to examine the potential benefit of implementing UPS-EP in terms of dialysis costs.

Results: The majority of UPS patients could receive UPS-EP (214/270 patients) and were able to make a decision (177/214), although not all patients received their preferred choice (159/177). Regression analysis demonstrated that the initial dialysis modality was a predictive factor for referral and receipt of UPS-EP and modality decision making. In contrast, age was a predictor for referral and receipt of UPS-EP only, and comorbidity was not a predictor for any step, except for myocardial infarction, which was a weak predictor for lower likelihood of receiving preferred modality. Country practices predicted UPS-EP receipt and decision making. Economic analysis demonstrated the potential benefit of UPS-EP implementation because dialysis modality costs were associated with modality distribution driven by patient preference.

Conclusion: Education and decision support can allow UPS patients to understand their options and choose dialysis modality, and attention needs to be focused on ensuring equity of access to educational programs, especially for the elderly. Physician practice and culture across units/ countries is an important predictor of UPS patient management and modality choice independent of patient-related factors. Additional work is required to understand and improve patient pathways to ensure that modality preference is enacted. There appears to be a cost benefit of delivering education, supporting choice, and ensuring that the choice is enacted in UPS patients.

Keywords: chronic kidney disease, dialysis, patient education, unplanned dialysis start, decisionmaking process

Correspondence: Anna Machowska Division of Renal Medicine and Baxter Novum, Department of Clinical Science, Intervention, and Technology, Karolinska Institutet, M99, Karolinska University Hospital, Huddinge, SE-I4I 86 Stockholm, Sweden

Email anna.machowska@ki.se

\section{Introduction}

The prevalence of chronic kidney disease (CKD) has reached epidemic proportions, with $10 \%-12 \%$ of the population and $50 \%$ of elderly showing signs of kidney dysfunction. ${ }^{1} \mathrm{~A}$ proportion of CKD patients progressively lose kidney function until dialysis is required 
- such patients have a very high mortality risk ${ }^{2}$ and experience a life-changing impact on quality of life and functional status. ${ }^{3}$ Dialysis accounts for approximately $1 \%-2 \%$ of the health care expenditure in European countries. ${ }^{4}$

The process of transition from CKD to end-stage renal disease (ESRD) presents a significant challenge to patients and caregivers. ${ }^{5}$ Careful clinical management is crucial and several factors such as early referral to nephrologist, better coordination of medical care, management of CKD complications, and education around dialysis options that is based on informed consent help a patient commence dialysis in a planned way. It is important to prevent a disorganized, emergency unplanned dialysis start (UPS). UPS remains a worldwide concern and it is estimated that 24\%-49\% of patients commence dialysis in such a way. ${ }^{6}$ This group of patients is a higher burden to the dialysis units due to increased morbidity and mortality, ${ }^{7}$ increased use of health care resources, ${ }^{8}$ and, importantly, UPS patients are less likely to be educated, receive a choice of dialysis modality and choose a home dialysis therapy. UPS patients typically start and stay on in-center hemodialysis (HD), compared to planned dialysis ${ }^{9}$ patients who more often can choose home-based peritoneal dialysis (PD) or home HD. European guidelines ${ }^{10}$ state that all renal replacement therapy (RRT) centers should provide patients and their families with wellbalanced information about the different RRT modalities by means of a structured education program and this applies also to late referred and UPS patients. The education about dialysis options of UPS patients is a challenge for every dialysis unit, and it can be perceived that such patients with intense and urgent medical needs cannot be educated or make a choice. However, in single center studies, it has been shown to be possible, and that this results in increased incidence of PD choice. ${ }^{11}$ The Offering Patients Therapy Options in Unplanned Start (OPTiONS) was a multicenter observational study ${ }^{12}$ in Europe on the implementation of a UPS educational program (UPS-EP). USP-EP includes a specific educational program with decision support tools to promote shared decision making (SDM) within the context of a patient pathway improvement program.

In the current study, we aimed to investigate factors that may influence three key steps on the patient pathway in UPS patients within the OPTiONS study: 1) referral to and receiving UPS education, 2) making a decision about dialysis modality, and 3) receiving the preferred dialysis modality after decision making. In addition, we also aimed to evaluate the potential economic consequences of UPS-EP to determine if the investment to deliver the program is likely to be worthwhile.

\section{Methods}

\section{Study design and patient enrollment}

This was a noninterventional, prospective, multicenter, observational study of patients starting unplanned dialysis therapy. The study was approved by all appropriate Ethics Committees (Regionala etikprovningsnamnden I Stockholm, 2012/2:2; West Midlands MREC, UK 11/WM0160; Ethikkomission der Atrzekammer Nordrhein, 2011222; Ethikkomission der Atrzekammer Saarlandes, 127/11; Ethikkomission Tubingen, 376/2011B02; Ethikkomission der Atrzekammer, Stuttgart 1816/BX; Ethikkomission der Bayerischen, Munich 11068; Ethikkomission der Medizinischen Universitat Wien, Vienna 605/2011; CNIL, Paris EGY/ABE/AR122444; CNOM, Paris FR/IH/SRMI/CN-11-349-117; CCTIRS, Paris 11.688) and was conducted in adherence to the Declaration of Helsinki after written informed consent was obtained from each individual. Participating centers had implemented the UPS-EP into their routine clinical practice. Twenty-six centers in six European countries (Austria [AU], Denmark [DK], Germany [DE], France [FR], Sweden [SE], and the UK) recruited all UPS patients presenting in their units and followed them up for 12 months. The inclusion criteria were wide to capture all UPS patients who were identified on clinical presentation and considered actively for education within the structured UPS-EP with the use of decision support tools. Although some UPS patients may be judged as clinically not suitable for this educational approach or would not be able to make a modality choice due to medical reasons, they were still identified and included in the overall UPS cohort. The patients included had CKD stage 5, were aged between 18 and 90 years at the time when informed consent was signed, and commenced dialysis in an unplanned way on the basis of clinical criteria of presentation to the nephrologist within 1 month of needing dialysis (as previously "unknown" ESRD patient) or who were being followed by a nephrologist but requiring urgent dialysis commencement by central venous catheter for HD or an acutely placed PD catheter.

Patients could receive the UPS-EP at the time of presentation or following dialysis start, as judged by clinical assessment.

\section{The UPS-EP approach}

The UPS-EP was developed in an attempt to modulate the UPS patients' pathway and allow dialysis modality education and modality decision making in UPS patients. The program is composed of an education program, together with an examination and optimization of the flow of UPS patients in renal care units. The program was developed in collaboration with five European dialysis units linked to academic institutions 
specialized in patient education. The program is focused on facilitating the decision-making process for patients choosing chronic RRT. There is no consensus regarding the structure and content for dialysis modality decision making, although standards have been suggested for planned start CKD education programs. ${ }^{13}$ Programs tend to focus on general knowledge about CKD and its treatment, as well as on dialysis modality decisions. ${ }^{14}$ In contrast, the UPS-EP educational element focuses on the modality decision itself since this is the critical element of education/health literacy at this time of UPS presentation. This approach is confirmed in the oncology literature, ${ }^{15,16}$ wherein at the time of diagnosis, patients are found to be in need of information on treatment options and the future, and they need to get an individual picture of the impact of the disease and the treatment options. Thus, the UPS-EP included information on $\mathrm{HD}, \mathrm{PD}$, home $\mathrm{HD}$, and conservative care, as well as transplantation. The UPS-EP was delivered to the patients during at least three individual sessions by nurses using motivational interviewing methodology, at a pace determined by the educational nurse with assessment of the clinical condition. Educational material included a dialysis options booklet matching the educational material delivered by the nurse; a photograph-based book showing PD, HD, and home HD; and a unit-specific video of the techniques, alongside visits to the HD unit and demonstration of PD. It should be remembered that the majority of UPS patients would be receiving UPS-EP after commencing dialysis. In addition, decision support tools were used as key elements of UPS-EP, whereas their use in CKD education at the time of commencement of OPTiONS was minimal. Three aids were available to the centers, with the educators choosing for individual patients from the Ottawa online decision aid, a self-completion balance scale, and a set of decision cards that allowed the patient to prioritize the value to them of specific issues and factors related to dialysis modalities. The detailed description of the program was presented previously elsewhere. ${ }^{12}$ UPS-EP tools are presented in Figure 1 (Swedish version of the educational package).

Depending on the clinical condition of the UPS patient at the referral time to the nephrology unit in the participating center, the patient was directed to UPS-EP. This pathway has three key steps, which also comprise the structure of our analysis:

1. Referral to and receipt of an educational program conducted by an identified educator;

2. Decision making about a dialysis modality after education with the assistance of decision support tools;

3. Enacting the choice, including the process, to ensure timely access to permanent dialysis and other procedures related to providing patients with the preferred dialysis technique.

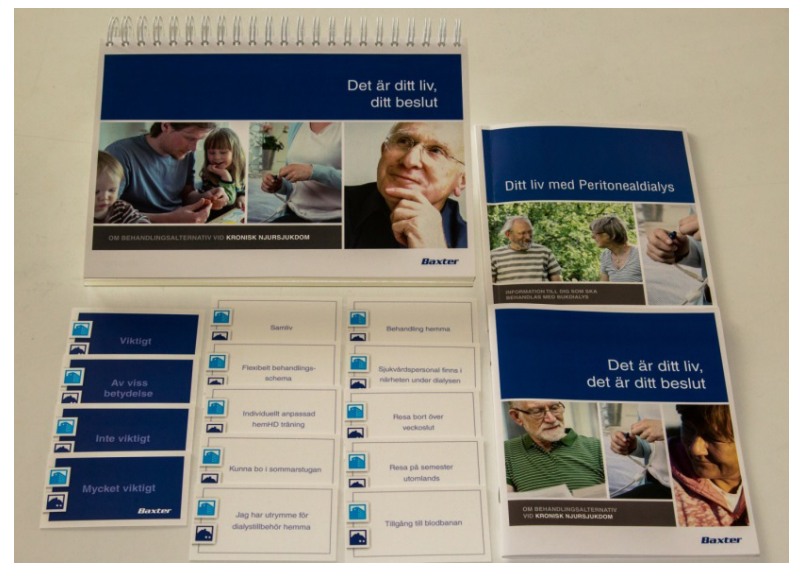

Figure I UPS-EP tools (Swedish version).

Abbreviations: UPS, unplanned dialysis start; EP, educational program.

The number of patients receiving and completing the program and the number of patients making a modality decision, together with the final dialysis modality chosen (PD or HD), were measured. During the 12-month follow-up period, the actual number of patients receiving their chosen modality was recorded, together with their clinical outcomes - these primary outcome data are presented elsewhere (Machowska et al, unpublished data, 2016).

\section{Economic consequences of UPS-EP}

This is not a complete economic analysis looking at all health care resource utilization; however, we aimed to examine the broad economic consequences of UPS-EP to confirm or deny the potential benefits of investing in such a program. The estimates of the dialysis costs were based on the published literature. Annual per patient cost for PD is estimated to be $€ 30,000 .{ }^{17}$ Across Europe, $\mathrm{HD}$ is 1.41 times $^{18}$ more expensive than $\mathrm{PD}$, ie, annual per patient cost is $€ 42,300$. Therefore, we examined the potential modality costs of the 177 patients who completed UPS-EP and made a choice according to, 1) their preferred choice of modality, and 2) the actual dialysis modality received by these patients. In addition, we estimated the potential dialysis costs considering the historical rate of $10 \%$ of the same number of UPS patients who would receive PD in usual clinical practice. ${ }^{11}$

\section{Statistical analysis}

Data are expressed as median, percentage, or odds ratio (OR; with $95 \%$ confidence interval $[\mathrm{CI}]$ ), as appropriate. Statistical significance was set at the level of $P<0.05$. Logistic regression was performed to investigate the factors that may influence the key three steps in the education pathway: 1) receiving education, 2) making dialysis modality choice, and 3) receiving modality according to patient preferences. Depending on the 
model the following explanatory variables were included: age, gender, presence of diabetes, congestive heart failure (CHF), time between first referral to nephrologists and first dialysis session (categorized as needing dialysis at the referral day, or later), initial dialysis modality (modality to which patient was assigned by the physician), center (countries were divided into two groups based on historical home dialysis use: FR, DE, and AU formed one group; the UK, SE, and DK comprised the second group), and patient source (inpatient/outpatient admission as a proxy for timing of the education). Statistical analyses were performed using the statistical software SAS version 9.4 (SAS Campus Drive, Cary, NC, USA).

\section{Results}

\section{Characteristics of UPS patients at inclusion and their educational pathway}

Two hundred and seventy patients $(n=270)$ were successfully enrolled in the OPTiONS Registry, which evaluated the effect of the UPS-EP on the dialysis modality choice made by UPS patients. Patients showed, at inclusion, characteristics typical for patients with ESRD commencing dialysis in Europe. ${ }^{19}$ The median age was 69 (10th-90th percentiles: 40-83) years and $64 \%$ were males. Diabetes (41\%), CHF (31\%), myocardial infarction (18\%), and peripheral vascular disease (18\%) were the most prevalent comorbidities. The majority of the patients were from inpatient admission (71\%) and were referred to the nephrology unit equally often from primary care and from other hospital specialties. Despite our patients starting dialysis in an unplanned manner, only $17 \%$ of patients needed dialysis on the referral day, $32 \%$ of patients received their first dialysis between 2 days and 2 weeks, whereas $15 \%$ got first dialysis between 2 weeks and 3 months, and 36\% received it after 3 months or later.

The majority of UPS patients $(n=214)$ were medically suitable for either dialysis modality and received UPS-EP whereas 56 patients never received education. The majority of patients completed UPS-EP ( $\mathrm{n}=203)$, and 177 patients were able to make a decision on dialysis modality. Among those who made a decision on initial modality, 103 patients chose PD and 74 HD.

There was still a group of patients following UPS-EP who did not make, or were unable for clinical reasons to make, a decision on a preferred dialysis therapy. In addition, not all patients received the modality that they chose after education and supported decision making. Out of 103 patients who chose $\mathrm{PD}$, only 89 patients received it, and among 74 patients who chose $\mathrm{HD}, 70$ patients received $\mathrm{HD}$ according to their recorded decision (Figure 2).

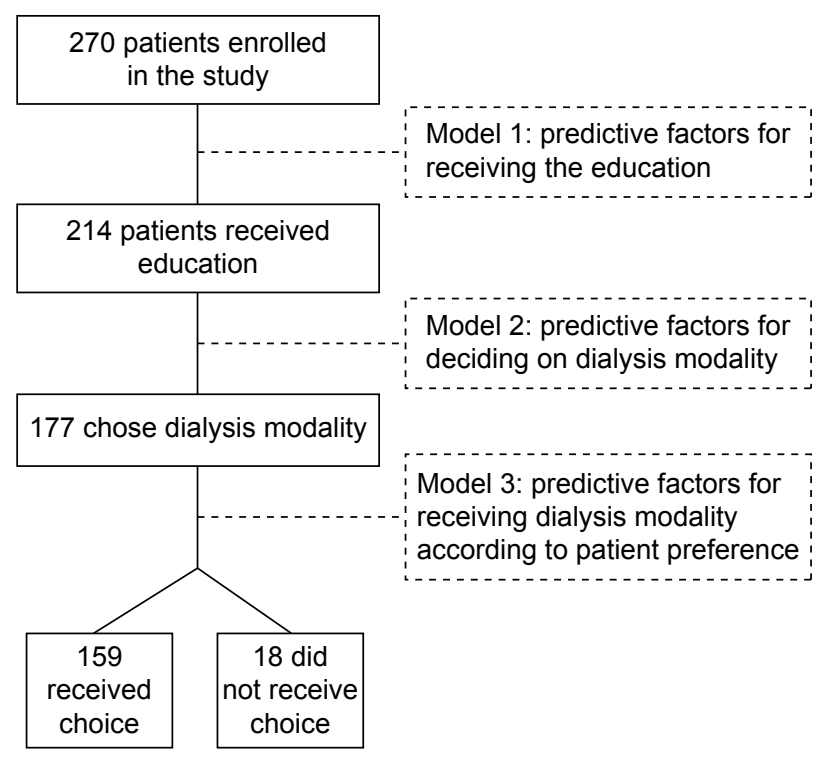

Figure 2 Regression models for the factors influencing the three key steps of USP-EP. Abbreviations: UPS, unplanned dialysis start; EP, educational program.

The UPS-EP is being integrated into a complex care pathway and needs to be completed and acted upon in a timely manner to assist in the management of this life-threatening condition. This study aimed to recruit all UPS patients and, therefore, we can examine the factors that may predict the following three key steps:

1. Receiving UPS-EP;

2. Decision making;

3. Enacting choice.

\section{Application of regression models to assess factors influencing education and choice}

A useful application of regression models has been developed for the purpose of this study. By applying this technique, we were able to address the question about the possible factors influencing the three key steps of the UPS-EP education pathway (Figure 2). The results of the analysis are presented in the following sections.

\section{Factors influencing receipt of UPS-EP}

We investigated the factors influencing receipt of UPS-EP in the 270 UPS patients enrolled in the study (Table 1). Logistic regression analysis showed that patients who were older than 69 years had $60 \%$ lower chance of receiving education: OR $=0.40$ (CI: $0.20-0.80)$. OR for receiving education was significantly higher among patients with $\mathrm{PD}$ as an initial dialysis modality $(\mathrm{OR}=4.81 ; \mathrm{CI}: 1.85-12.50)$. Moreover, country was a significant predictor of receiving education; patients in $\mathrm{DE}$, 
Table I Logistic regression analysis evaluating the predictors of receiving education in 270 UPS patients

\begin{tabular}{|c|c|c|}
\hline Variables & Odds ratio $(95 \% \mathrm{Cl})$ & P-value \\
\hline Age, $\geq 69$ years & $0.40(0.20-0.80)$ & 0.01 \\
\hline Gender, male versus female & $0.73(0.37-1.45)$ & 0.37 \\
\hline Admission, inpatients versus outpatients & $0.59(0.27-1.31)$ & 0.20 \\
\hline Diabetes, presence versus absence & $0.65(0.33-1.28)$ & 0.21 \\
\hline Time between first referral to nephrologists and first dialysis, the same day versus later & $0.63(0.28-1.42)$ & 0.26 \\
\hline Initial dialysis modality, PD versus HD & $4.81(1.85-12.50)$ & 0.001 \\
\hline Congestive heart failure & $0.71(0.34-1.49)$ & 0.37 \\
\hline Country, DE + FR + AU versus the UK + SE + DK & $0.38(0.18-0.82)$ & 0.01 \\
\hline
\end{tabular}

Notes: Pseudo- $r^{2}=0.12$. Significant values are marked in bold $(P<0.05)$.

Abbreviations: AU, Austria; Cl, confidence interval; DE, Germany; DK, Denmark; FR, France; HD, hemodialysis; PD, peritoneal dialysis; SE, Sweden; UPS, unplanned dialysis start.

FR, and AU had markedly lower chances of receiving education compared to patients from the UK, SE, and DK: $\mathrm{OR}=0.38$ (CI: 0.18-0.82). Nonsignificance of comorbid illnesses such as diabetes and CHF means that these conditions were not discriminating factors for receiving an education.

\section{Factors influencing dialysis modality decision-making process}

We aimed to evaluate the factors that may influence the decision-making pathway in patients starting unplanned dialysis (Table 2). Only two factors, namely, starting dialysis modality and country, predicted decision making. Logistic regression model analysis shows that patients starting on PD as an initial dialysis modality (OR $=6.33 ; \mathrm{CI}: 2.89-13.87)$ had significantly higher chances of making a choice. Furthermore, country category was shown to be a statistically significant predictor of making a decision about dialysis modality: patients from DE, FR, and AU had smaller chances of making a choice than patients from the UK, $\mathrm{SE}$, and $\mathrm{DK}(\mathrm{OR}=0.50$; CI: 0.28-0.87).

\section{Factor influencing receipt of dialysis therapy according to patient preferences}

Regardless of the ability to make a dialysis modality decision after receiving education, there were still patients who did not receive the dialysis option according to the informed choice.

The logistic regression analysis shows that the selected factors, such as age, gender, and presence of diabetes, are not significant predictors for patients to receive their preferred dialysis option. Initial dialysis modality just failed to reach statistical significance. The only significant predictor was history of myocardial infarction ( $\mathrm{OR}=0.15$; CI: $0.05-0.48)$; thus, patients with myocardial infarction were less likely to receive their preferred choice of final modality (Table 3).

\section{Economic evaluation}

Chronic dialysis is an expensive therapy, and the annual costs for consumables, staff, infrastructure, and transport are highly variable between countries. We aimed to perform a simple analysis of the annual dialysis costs to assess the potential economic impact of UPS-EP.

This is not a full health economic analysis of health care utilization but a hypothetical example providing a broad picture of dialysis costs only in relation to education and decision making. We examined the modality costs of the 177 patients who completed UPS-EP and made a choice, according to the following factors:

1. Their preferred choice of modality;

2. The actual dialysis modality received by patients;

Table 2 Logistic regression analysis evaluating the predictors of making the choice on dialysis modality after receiving education in 214 UPS patients

\begin{tabular}{lll}
\hline Variables & Odds ratio (95\% Cl) & $P$-value \\
\hline Age, $\geq 69$ years & $0.64(0.37-I . I I)$ & 0.1 I \\
Gender, male versus female & $1.70(0.97-2.98)$ & 0.07 \\
Diabetes, presence versus absence & $0.94(0.53-1.64)$ & $0.8 I$ \\
Initial dialysis modality, PD versus HD & $6.33(2.89-13.87)$ & $<0.001$ \\
Country, DE + FR + AU versus the UK + SE + DK & $\mathbf{0 . 5 0 ( 0 . 2 8 - 0 . 8 7 )}$ & $\mathbf{0 . 0 2}$ \\
\hline
\end{tabular}

Notes: Pseudo- $r^{2}=0.12$. Significant values are marked in bold $(P<0.05)$.

Abbreviations: AU, Austria; Cl, confidence interval; DE, Germany; DK, Denmark; FR, France; HD, hemodialysis; PD, peritoneal dialysis; SE, Sweden; UPS, unplanned dialysis start. 
Table 3 Logistic regression analysis evaluating the predictors of receiving dialysis modality according to patient preferred choice $(n=159)$ versus not receiving preferred dialysis modality according to patient choice $(n=18)$ following completion of UPS-EP

\begin{tabular}{lll}
\hline Variables & Odds ratio (95\% CI) & $\mathbf{P}$-value \\
\hline Age, $\geq 67$ years & $0.98(0.32-2.97)$ & 0.95 \\
Gender, male versus female & $0.96(0.28-3.24)$ & 0.95 \\
Diabetes, presence versus absence & $0.84(0.28-2.5 I)$ & 0.76 \\
Myocardial infarction & $\mathbf{0 . 1 5 ( 0 . 0 5 - 0 . 4 8 )}$ & $\mathbf{0 . 0 0 I}$ \\
Initial dialysis modality, PD versus HD & $3.66(0.97-13.88)$ & 0.06 \\
\hline
\end{tabular}

Notes: Pseudo- $r^{2}=0.07$. Significant values are marked in bold $(P<0.05)$.

Abbreviations: $\mathrm{Cl}$, confidence interval; $\mathrm{EP}$, educational program; $\mathrm{HD}$, hemodialysis; $\mathrm{PD}$, peritoneal dialysis; UPS, unplanned dialysis start.

3. The historical group of $10 \%{ }^{11}$ of UPS patients who would choose PD with no education (usual care).

Table 4 shows the modality costs in 177 patients according to their preferred choice of modality and according to the actual modality received (total cost: $€ 6,220,200$ versus $€ 6,343,200$ ). However, it is important to note that standard UPS care results in typically only $10 \%$ of patients receiving PD. ${ }^{11}$ Therefore, the estimated modality costs if only $10 \%$ of these 177 have received PD demonstrates the potential cost savings of implementing dialysis choice by the UPS patients through UPS-EP (total cost: $€ 6,343,200$ versus $€ 7,265,700)$.

\section{Discussion}

The OPTiONS study focused on patients presenting with ESRD and the need to commence chronic dialysis. These patients require rapid clinical decision making, significant medical intervention, and psychosocial support during this period as they come to terms with their future. In the midst of this period, dialysis is commenced, but, most frequently, ${ }^{20}$ a choice of dialysis is not routinely provided. Therefore, most patients receive and remain on in-center HD and do not receive a choice of home dialysis. The UPS-EP implemented in the OPTiONS study was a tailored educational package within the context of a clinical pathway approach to improve the management of UPS. The study demonstrated that it was possible for most patients to be referred to and receive education as well as make decisions, but problems remain with overall clinical management. The observations can be examined in the context of the previous nephrology literature on UPS as well as oncology literature since the cancer diagnosis and initial clinical pathway follow similar patterns as in ESRD - an unexpected diagnosis, need for medical interventions, and education requirements for informed choices in the context of a life-threatening disease. In the following paragraphs, we discuss the outcome of the OPTiONS study in the following three areas:

1. Which patients are referred to and are suitable for education?

2. Can decision making be supported?

3. What are the challenges around enacting patient choice? OPTiONS aimed to include all UPS patients and not only a selected group who professionals deemed to be appropriate for education. The majority of UPS patients were found to be suitable for referral to UPS-EP, demonstrating that even this complex group of patients can be educated. The UPS-EP, which focuses on modality choice, appears to meet the needs of health care professionals who can see the benefits of referral. It is interesting to note that UPS patient management has a multidisciplinary focus and that recently ${ }^{15,21}$ it has been noted in a study of health care professionals programs that while oncology nurses stress educational content relating to patient problems in their everyday life, oncology physicians focus more on informed consent and the patients' right to information. UPS education programs need to meet all these requirements as well as facilitate health literacy of patients. Health literacy is important in terms of dialysis modality decision making, but this is a challenge in practice. In a recent study of CKD patients progressing to a planned start, ${ }^{22}$ low levels of health literacy were observed 2 months after education, with $38 \%$ of patients unsure about how kidney disease was to be treated. It is clear not only that health literacy varies, but also that dissociation exists between the perceived and the measured literacy of patients. ${ }^{23}$ Another recent study ${ }^{24}$ confirmed that limited health literacy is common $(10 \%-50 \%$ of CKD patients) and was more frequent in those with less formal education, men, and patients of nonwhite racial origin. Of interest to the current study, these authors describe the

Table 4 Dialysis modality costs according to preferred modality choice following UPS $(n=177)$, actual modality received by these patients, and calculated costs derived for usual care modality distribution

\begin{tabular}{llll}
\hline Modality distribution & PD cost $(\boldsymbol{\epsilon})$ & HD cost $(\boldsymbol{\epsilon})$ & Total cost $(\boldsymbol{\epsilon})$ \\
\hline I77 patients according to their preferred choice of modality & $3,090,000$ & $3,130,200$ & $6,220,200$ \\
I 77 patients according to actual modality received & $2,790,000$ & $3,553,200$ & $6,343,200$ \\
I77 patients according to usual care & 540,000 & $6,725,700$ & $7,265,700$ \\
\hline
\end{tabular}

Abbreviations: $\mathrm{HD}$, hemodialysis; PD, peritoneal dialysis; UPS, unplanned dialysis start. 
importance of "organizational health literacy", where a unit should have systems and processes in place with an ethos of SDM and material appropriate for the educational needs of patients. This summarizes the UPS-EP approach to organizational focus, which allowed most UPS patients, despite the mean age of almost 70 years and significant comorbidity, to be referred for education. OPTiONS approach has demonstrated some factors that are predictive of education referral and therefore require attention. Although comorbidity issues were not relevant, age was a predictive factor, with the elderly patients being less likely to be referred for education. It is known that elderly patients ${ }^{25}$ have good clinical outcomes with PD and age is not a barrier to choice of PD; indeed, elderly patients may see particular benefits in a home dialysis therapy. Age has been seen to be a factor in patient education in other disease areas. Thus, a study in cardiac failure patients found that older age was associated with lower health literacy. In oncology, a study of colorectal cancer patients ${ }^{26}$ showed that inadequate functional health literacy was more frequently observed in patients with age over 65 years. An interesting finding was observed in a large study of 2,750 colorectal cancer patients in Ireland: ${ }^{27}$ older patients were more likely to have an emergency presentation, analogous to UPS in that study, and emergency patients had worse clinical outcomes. Age is also relevant in determining the focus of educational areas for oncology patients, ${ }^{16}$ and delivering education to more elderly patients is feasible. A study ${ }^{28}$ examined age as a factor that could explain the regional variation noted in the use of two surgical options for breast cancer. Although older age was associated with less knowledge of the disease and its therapy, the frequency of patient-based decisions did not vary much and there was no difference in the distribution of the two surgical options. This last study of regional variation does raise an issue also observed in this study - the country of the study site was a predictor of the likelihood to receive education in UPS. The geographical variation in PD use across Europe is well known and has not changed in the past 20-30 years. ${ }^{29}$ This appears to reflect physician belief and practice patterns, which are also playing out within the context of the current study. Additional work is needed to modulate patient pathways and patient centricity of the professionals. In contrast, our observation that the type of initial dialysis access is a predictor of receiving education perhaps reflects the opposite - physicians who firmly believed in patient choice and home dialysis with PD are more likely to commence with PD while educating and assisting in decision making. A UPS-EP can be implemented in practice, but more work is needed to ensure equity of access for the elderly and to continue understanding physician attitudes and practices, which influence education referral.

OPTiONS results show that not only can most UPS-EP patients receive education but that decision support tools as part of the program may help most patients to make a decision. In addition, there were no patient demographic factors that predict ability to make a decision; in particular, age and comorbidity are not influential. Thus, these factors should not be used as part of value judgments by clinical teams to assume which patients can or cannot make decisions; with the correct information and decision support, decision making is possible. The benefits of decision support tools in terms of improving knowledge and reducing decisional conflict have been summarized in a recent systematic review. ${ }^{30}$ In the past 3 years, decision support tools have been developed for planned start patients with $\mathrm{CKD}^{31}$ and applied in clinical practice. OPTiONS has shown that the UPS-EP allows most patients to make a decision; the wide inclusion criteria of the study allowed almost all UPS patients to be included and allowed us to examine predictive factors for decision making. Once again, the predictive factors relate to the country and the original access route used for the first dialysis session. There are geographical differences in overall patient centricity of physicians in clinical practice across other disease areas. ${ }^{32}$ In addition, simulation studies on dialysis modality decision have shown that physicians from different countries do make different clinical decisions over specific clinical cases. Physicians should be aware of their own approach to uncertainty and how this might influence UPS patients' responses when engaging in SDM. ${ }^{33}$ There is a need to consider how to assist physicians to move into a more SDM style and avoid the need to be unnecessarily "in control". 34

However, the final step of UPS-EP is ensuring that the preferred choice is enacted, and this is still a problem - in particular, for patients wishing to switch to PD. It is encouraging that age was not predictive of failure to receive preferred modality, although a past history of myocardial infarction was a weak predictor. This may be a reflection of clinical assessment of individual patients' cardiac function or cardiovascular risk and the impact of PD upon it. The initial dialysis modality still seems a relevant factor, but its predictive value just failed to reach statistical significance. Overall, the predictive power and significant factors influencing the enactment of patient preferred choice speaks to remaining cultural, pathway of care, or logistic challenges, which require further examination in order to use further interventions in clinical practice following patient education and decision making to ensure modality choice progresses. There may be long-term 
adverse clinical and patient-reported outcomes associated with this failure to enact the decision as patients get frustrated with a dialysis modality they did not prefer. Full examination of a unit's UPS pathway is critical to determine the timing of education, assess competency of the educator, and smoothen the patient's path to the preferred modality. An interesting analogous situation can be seen in oncology, whereby understanding the remainder of the pathway of care for decision making in prostate cancer found little benefit of a specific education and decision support intervention due to timing and other interventions from surgeons. ${ }^{35}$ Pathway intervention can be beneficial, as confirmed by this study, and a specific pathway-focused Renal Triage Nurse reviewing all patients commencing dialysis in a suboptimal way allowed $28 \%$ of patients to switch from HD to a home therapy after education and coordination of their care following decision making to enact the decision. ${ }^{36}$

Chronic dialysis is expensive and there is growing concern over the economic impact of suboptimal UPS of dialysis on health care systems. Canadian data showed that the average total cost per patient was an estimated $\$ 63,225$ for the suboptimally initiated patients and $\$ 39,260$ for the optimally (dialysis initiation as an outpatient, using arteriovenous fistula, graft, or PD catheter) initiated patient. ${ }^{37}$ Most developed countries can provide PD at lesser expense to the health care system than HD. ${ }^{18}$ The brief assessment of dialysis modality costs for the 177 patients who completed UPS-EP and made a decision shows that patients who got their preferred choice had the lowest costs. Most expensive was if that same group of patients had received standard care with only around $10 \%$ receiving PD. ${ }^{11}$ But there is also a cost benefit of solving both the education/ decision making steps and the enacting patient modality choice steps. At the minimum, the investment in UPS-EP educational material and training nurse(s) would appear to be beneficial.

In summary, this analysis of the OPTIONS study has shown the overall clinical outcomes and potential cost benefits of implementing the UPS-EP and also shown that older age reduces the probability of a UPS patient receiving education but not the chance of making a modality decision. Other patient factors were found to neither influence education receipt nor the ability to make a dialysis modality decision. Cultural and country factors have a strong influence on the probability of both receiving education and making a decision. A small number of patients, mostly those wishing to switch from HD to PD, do not switch, so additional health care process factors need attention.

\section{Acknowledgments}

We thank all patients included in the study and all physicians, nurses, and other health care personnel who participated in the clinical care and data collection. This observational study, Offering Patients Therapy Options in Unplanned Start (OPTiONS), was supported by a grant from Baxter Healthcare Corporation. Anna Machowska was funded by the European Union Marie Curie Initial Training Network grant (number FP7-PEOPLE-2011-ITN) for the project European Training and Research in Peritoneal Dialysis (EuTRiPD). This study was also supported by a grant from Baxter Healthcare Corporation to Karolinska Institutet.

\section{Disclosure}

Bengt Lindholm and Anna Machowska are employed by Baxter Healthcare Corporation. Peter Rutherford was employed by Baxter Healthcare Corporation at the time of the study and is now employed by Quintiles. The authors report no other conflicts of interest in this work.

\section{References}

1. Stenvinkel P. Chronic kidney disease: a public health priority and harbinger of premature cardiovascular disease. J Intern Med. 2010; 268(5):456-467.

2. Go AS, Chertow GM, Fan D, McCulloch CE, Hsu CY. Chronic kidney disease and the risks of death, cardiovascular events, and hospitalization. N Engl J Med. 2004;351(13):1296-1305.

3. Wasserfallen JB, Halabi G, Saudan P, et al. Quality of life on chronic dialysis: comparison between haemodialysis and peritoneal dialysis. Nephrol Dial Transplant. 2004;19(6):1594-1599.

4. Laplante S, Vanovertveld P. A renal policy and financing framework to understand which factors favour home treatments such as peritoneal dialysis, progress in peritoneal dialysis. In: Krediet R, editor. Progress in Peritoneal Dialysis. Rijeka: InTech; 2011:115-132.

5. Amedia CA, Perazella MA. Transition of patients from chronic kidney disease to end-stage renal disease: better practices for better outcomes. Dis Manag. 2006;9(6):311-315.

6. Mendelssohn DC, Malmberg C, Hamandi B. An integrated review of "unplanned" dialysis initiation: reframing the terminology to "suboptimal" initiation. BMC Nephrol. 2009;10:22.

7. Metcalfe W, Khan IH, Prescott GJ, Simpson K, MacLeod AM. Can we improve early mortality in patients receiving renal replacement therapy? Kidney Int. 2000;57(6):2539-2545.

8. Górriz JL, Sancho A, Pallardó LM, et al. [Prognostic significance of programmed dialysis in patients who initiate renal substitutive treatment. Multicenter study in Spain]. Nefrologia. 2002;22(1):49-59.

9. Marrón B, Ortiz A, de Sequera P, et al. Impact of end-stage renal disease care in planned dialysis start and type of renal replacement therapy - a Spanish multicentre experience. Nephrol Dial Transplant. 2006;21(suppl 2):ii51-ii55.

10. Covic A, Bammens B, Lobbedez T, et al. Educating end-stage renal disease patients on dialysis modality selection: clinical advice from the European Renal Best Practice (ERBP) Advisory Board. Nephrol Dial Transplant. 2010;25(6):1757-1759.

11. Rioux JP, Cheema H, Bargman JM, Watson D, Chan CT. Effect of an in-hospital chronic kidney disease education program among patients with unplanned urgent-start dialysis. Clin J Am Soc Nephrol. 2011;6(4): 799-804. 
12. Machowska A, Alscher MD, Vanga SR, Koch M, Aarup M, Rutherford P. Offering patients therapy options in unplanned start: development and implementation of an education program for unplanned-start patients. Adv Perit Dial. 2015;31:69-73.

13. Isnard Bagnis C, Crepaldi C, Dean J, et al. Quality standards for predialysis education: results from a consensus conference. Nephrol Dial Transplant. 2015;30(7):1058-1066.

14. Van den Bosch J, Warren DS, Rutherford PA. Review of predialysis education programs: a need for standardization. Patient Prefer Adherence. 2015;9:1279-1291.

15. Heli VR, Helena LK, Liisa I, Kimmo L, Heikki M. Oncologic patients' knowledge expectations and cognitive capacities during illness trajectory: analysis of critical moments and factors. Holist Nurs Pract. 2015;29(4):232-244.

16. O’Leary KA, Estabrooks CA, Olson K, Cumming C. Information acquisition for women facing surgical treatment for breast cancer: influencing factors and selected outcomes. Patient Educ Couns. 2007; 69(1-3):5-19.

17. Liu FX, Quock TP, Burkart J, Noe LL, Inglese G. Economic evaluations of peritoneal dialysis and hemodialysis: 2004-2012. F1000Research. 2013;2:273.

18. Karopadi AN, Mason G, Rettore E, Ronco C. Cost of peritoneal dialysis and haemodialysis across the world. Nephrol Dial Transplant. 2013;28(10):2553-2569.

19. ERA-EDTA Registry. ERA-EDTA Registry Annual Report 2012. Amsterdam: ERA-EDTA Registry; 2014. Available from: https://www. era-edta-reg.org/index.jsp?p=14. Accessed October 19, 2016.

20. Ghaffari A. Urgent-start peritoneal dialysis: a quality improvement report. Am J Kidney Dis. 2012;59(3):400-408.

21. Vaartio-Rajalin H, Huumonen T, Iire L, et al. Patient education process in oncologic context: what, why, and by whom? Nurs Res. 2015;64(5): 381-390.

22. Gray NA, Kapojos JJ, Burke MT, Sammartino C, Clark CJ. Patient kidney disease knowledge remains inadequate with standard nephrology outpatient care. Clin Kidney J. 2016;9(1):113-118.

23. Nesrallah GE, Mendelssohn DC. Reflections on education interventions and optimal dialysis starts. Perit Dial Int. 2013;33(4): 358-361.

24. Dageforde LA, Cavanaugh KL. Health literacy: emerging evidence and applications in kidney disease care. Adv Chronic Kidney Dis. 2013;20(4): 311-319.
25. Brown EA, Johansson L, Farrington K, et al. Broadening Options for Long-term Dialysis in the Elderly (BOLDE): differences in quality of life on peritoneal dialysis compared to haemodialysis for older patients. Nephrol Dial Transplant. 2010;25(11):3755-3763.

26. Busch EL, Martin C, DeWalt DA, Sandler RS. Functional health literacy, chemotherapy decisions, and outcomes among a colorectal cancer cohort. Cancer Control. 2015;22(1):95-101.

27. Comber H, Sharp L, de Camargo Cancela M, Haase T, Johnson H, Pratschke J. Causes and outcomes of emergency presentation of rectal cancer. Int J Cancer. 2016;139(5):1031-1039.

28. Bleicher RJ, Abrahamse P, Hawley ST, Katz SJ, Morrow M. The influence of age on the breast surgery decision-making process. Ann Surg Oncol. 2008;15(3):854-862.

29. Machowska A, Rutherford P. Peritoneal dialysis use within the context of the population and healthcare systems of Europe - differences, trends and future challenges. Int J Artif Organs. 2016;39(5):211-219.

30. Stacey D, Légaré F, Col NF, et al. Decision aids for people facing health treatment or screening decisions. Cochrane Database Syst Rev. 2014;1:CD001431.

31. University of Leeds [webpage on the Internet]. Yorkshire Dialysis Decision Aid. 2016. Available from: http://www.yodda.leeds.ac.uk/ Survey/Introduction. Accessed September 26, 2016.

32. Davis K, Stremikis K, Schoen C, Squires D. Mirror, mirror on the wall, 2014 update: how the US health care system compares internationally. Commonw Fund. 2014;16:6-30.

33. Politi MC, Clark MA, Ombao H, Légaré F. The impact of physicians' reactions to uncertainty on patients' decision satisfaction. J Eval Clin Pract. 2011;17(4):575-578.

34. Mudge S, Kayes N, McPherson K. Who is in control? Clinicians' view on their role in self-management approaches: a qualitative metasynthesis. BMJ Open. 2015;5(5):e007413.

35. Berry DL, Wang Q, Halpenny B, Hong F. Decision preparation, satisfaction and regret in a multi-center sample of men with newly diagnosed localized prostate cancer. Patient Educ Couns. 2012;88(2):262-267.

36. Hanko J, Jastrzebski J, Nieva C, White L, Li G, Zalunardo N. Dedication of a nurse to educating suboptimal haemodialysis starts improved transition to independent modalities of renal replacement therapy. Nephrol Dial Transplant. 2011;26(7):2302-2308.

37. Piwko C, Vicente C, Marra L, et al. The STARRT trial: a cost comparison of optimal vs sub-optimal initiation of dialysis in Canada. $\mathrm{J} \mathrm{Med}$ Econ. 2012;15(1):96-104
Patient Preference and Adherence

\section{Publish your work in this journal}

Patient Preference and Adherence is an international, peer-reviewed, open access journal that focuses on the growing importance of patient preference and adherence throughout the therapeutic continuum. Patient satisfaction, acceptability, quality of life, compliance, persistence and their role in developing new therapeutic modalities and compounds to optimize

\section{Dovepress}

clinical outcomes for existing disease states are major areas of interest for the journal. This journal has been accepted for indexing on PubMed Central. The manuscript management system is completely online and includes a very quick and fair peer-review system, which is all easy to use. Visit http://www. dovepress.com/testimonials.php to read real quotes from published authors. 